\title{
Publisher Correction: Quantifying transmission electron microscopy irradiation effects using two-dimensional materials
}

\author{
Toma Susi (iD, Jannik C. Meyer (iD) and Jani Kotakoski iD
}

Nature Reviews Physics (2019) https://doi.org/10.1038/s42254-019-0058-y Published online 14 May 2019

This article was originally published with an error in the main text and the caption of Figure 1 . The original sentence beginning, "The atomic vibrations have been described using the statistical distribution of out-of-plane velocities...", should have read: "The atomic vibrations have been described using the statistical distribution of out-of-plane velocities, which is characterized by its mean-square width $\overline{v_{z}^{2}} \ldots$. . In addition, in the caption of Figure 1, the original sentence beginning, "The distribution of out-of-plane velocities recorded for one carbon atom...", should have read: "The distribution of out-of-plane velocities recorded for one carbon atom (indicated by the red circle in panel b) once per femtosecond during a finite temperature simulation over 5 ps follows a normal distribution (panel c), the standard deviation (s.d.) of which is equal to the root-mean-square velocity $\left(\sqrt{v_{z}^{2}}\right)$ of the atom." This has now been corrected in the HTML and PDF version of the article.

We apologize to readers for this error.

https://doi.org/10.1038/s42254-019-0096-5 I Published online 31 July 2019 